\title{
Terahertz Focusing and Polarization Control in Large-Area Bias-Free Semiconductor Emitters
}

\author{
Joanna L. Carthy ${ }^{1}$ - Paul C. Gow ${ }^{2}$ Sam A. Berry ${ }^{2}$. \\ Ben Mills ${ }^{2}$. Vasilis Apostolopoulos ${ }^{1}$ (D)
}

Received: 12 September 2017 / Accepted: 31 October 2017/

Published online: 25 November 2017

(C) The Author(s) 2017. This article is an open access publication

\begin{abstract}
We show that, when large-area multiplex terahertz semiconductor emitters, that work on diffusion currents and Schottky potentials, are illuminated by ultrashort optical pulses they can radiate a directional electromagnetic terahertz pulse which is controlled by the angular spectrum of the incident optical beam. Using the lens that focuses the incident near-infrared pulse, we have demonstrated $\mathrm{THz}$ emission focusing in free space, at the same point where the optical radiation would focus. We investigated the beam waist and Gouy phase shift of the $\mathrm{THz}$ emission as a function of frequency. We also show that the polarization profile of the emitted $\mathrm{THz}$ can be tailored by the metallic patterning on the semiconductor, demonstrating radial polarization when a circular emitter design is used. Our techniques can be used for fast $\mathrm{THz}$ beam steering and mode control for efficiently coupling to waveguides without the need for $\mathrm{THz}$ lenses or parabolic mirrors.
\end{abstract}

Keywords Terahertz $\cdot$ Spectroscopy $\cdot$ Ultrafast lasers

\section{Introduction}

During the last two decades, there has been a lot of development on terahertz $(\mathrm{THz})$ emission mechanisms; however, methods for guiding and controlling the direction

Joanna L. Carthy and Paul C. Gow contributed equally to this publication

Vasilis Apostolopoulos

va2w07@soton.ac.uk

1 Physics and Astronomy, University of Southampton, SO17 1BJ, Southampton, UK

2 Optoelectronics Research Centre, University of Southampton, SO17 1BJ, Highfield,

Southampton, UK 
and polarization of $\mathrm{THz}$ radiation still require much refinement [1]. Specifically, the ability to direct and focus $\mathrm{THz}$ emission is necessary to maximize the potential of the $\mathrm{THz}$ technology for communications, imaging and medical technologies. Directing and focusing $\mathrm{THz}$ radiation would help applications in $\mathrm{THz}$ imaging by increasing the speed that images can be acquired. An integrated approach involving $\mathrm{THz}$ fibres is essential for industrial monitoring, communication applications and endoscopy, since free-space $\mathrm{THz}$ waves can easily be perturbed or attenuated [2, 3]. Furthermore, $\mathrm{THz}$ polarization and beam control would help to achieve efficient coupling in $\mathrm{THz}$ waveguiding systems where the $\mathrm{THz}$ must be launched upon a wire [4] or waveguide [5]. Methods that have been used in the $\mathrm{THz}$ regime for beam control include the following: programmable grating structures using cantilever arrays [1], optically gated semiconductors [6], difference frequency generators in phased arrays [7], translatable mirror elements [2], diffraction and optical hybrid setups [8, 9], nonlinear materials [10], photomixing [11] and optical pulse interference on a linear array of antennas [12]. Significantly, steering of photoconductive antennas (PCAs) has been achieved both optically and electrically. In the optical regime, standard PCA geometry was used to demonstrate that the THz electric field followed the path expected of the optical field [13]. Steering electrically was subsequently reported by creating an array of PCAs where the amplitude instead of phase was controlled [14-16].

For steering the $\mathrm{THz}$ beam of PCAs, it is necessary to fabricate complex electrode designs to achieve different polarization profiles while providing power to the electrodes and avoiding any electrical shorts, unlike the method we suggest here that requires little-to-no design alterations on the emitter. This paper explores beam steering using large-area emitters that generate $\mathrm{THz}$ radiation via diffusion currents, also known as the lateral photo-Dember (LPD) effect [17-19] and Schottky potentials [20, 21]. LPD and Schottky potential-based double metallic emitters have been found to have similar spectral bandwidth with photo-conductive emitters $[19,20]$. Various $\mathrm{THz}$ sources have been assessed for their capability to be implemented in a phase-delay array, but in-depth studies of a scheme using LPD emitters has yet to be tested.

We control the radiation of a THz LPD emitter by altering the characteristics of the input optical beam, transferring the properties of the excitation wavefront to the emitted $\mathrm{THz}$ wavefront to make the $\mathrm{THz}$ beam come to a focus at the point where the IR beam would. From our experiments, it was confirmed that focusing of the THz has been achieved and different $\mathrm{THz}$ wavelengths focus to larger or smaller beam waist sizes as expected by theory. Such a system is easily re-configurable and offers a broad level of optical control [22], the only component we need is a different lens to alter the focal point. Experimentally, depending on the position of our emitter, we characterize the $\mathrm{THz}$ beam waist and find that the emitted $\mathrm{THz}$ radiation undergoes a Gouy phase shift [23-26]. As the electric field is detected in THz-TDS, the Gouy shift manifests as a polarity flip in the THz detected wavefront if the emitter is placed after the focus of the lens [27], further supporting the notion that the $\mathrm{THz}$ is being focused in the same place as the optical pump. The importance and use of the Gouy shift has been reported in the field of THz imaging [28-36].

There is no electrical bias required for these devices, which are driven by diffusion currents and Schottky fields, so simplified metallic structures on the surface can be 
designed to achieve different polarization profiles for mode matching to waveguides. We demonstrate that different polarization profiles can be achieved through changing the metallic patterning on our devices. A circular device was fabricated and shown to exhibit a radial polarization profile, ideal for coupling to coaxial waveguides. Our techniques of focusing the $\mathrm{THz}$ radiation and controlling its polarization could be combined for $\mathrm{THz}$ beam steering, imaging and launching into a waveguide. This can be done with specially patterned emitters and with the help of an appropriate spatial light modulator to alter the phase profile of the optical pump beam.

\section{Fabrication of Emitters}

In all our experiments, $\mathrm{THz}$ was generated using a $2 \mathrm{~cm} \times 2 \mathrm{~cm}$ multiplexed doublemetal (DM) LPD emitter, fabricated using a double photolithography process and lift-off, with a pattern of partially overlapping $\mathrm{Au} / \mathrm{Pb}$ strips each with a thickness of $60 \mathrm{~nm}$ and width of $7 \mu \mathrm{m}$ (with a less than $5 \mathrm{~nm}$ layer of chromium to adhere the $\mathrm{Au}$ ) at a periodicity of $21 \mu \mathrm{m}$. The substrate material is semi-insulating GaAs. The fabrication and characterization of the emitters is described in [20, 21]. Two DM emitters with the above geometry were fabricated for the experiments, one with circular emitter elements to generate radial polarization and one emitter with straight elements that was used for the measurement of angular emission dependence and the focusing experiments.

\section{Angular Dependence of Emission and Radial Polarization}

The experimental data were taken with a standard THz-TDS setup as shown in Fig. 1. Excitation was performed using a mode-locked titanium sapphire laser emitting at $800 \mathrm{~nm}$, providing us with $\approx 100 \mathrm{fs}$ pulses at a repetition rate of $80 \mathrm{MHz}$ and peak pulse energy of approximately $1.25 \mathrm{~nJ}$. After the emitter, two THz TPX lenses of $\mathrm{f}=60 \mathrm{~mm}$ were used to collect all of the emitted radiation, and then to focus it upon the silicon lens aligned to behind the dipole of the detection emitter. Detection was performed using a LT-GaAs commercial antenna (MENLO Tera T8) and a lock in amplifier (with a response time of $100 \mathrm{~ms}$ ) measured the current changes at the detector. The emission dependence on emitter angle with respect to the pump beam was examined for the straight DM emitter and compared with that of a piece of bare semi-insulating GaAs. The schematic for this experiment is shown in Fig. 2a and the peak-to-peak $\mathrm{THz}$ emission with respect to emitter angle results are shown in Fig. 2b. At an angle of $0^{\circ}$ the SI-GaAs shows no detectable $\mathrm{THz}$ emission parallel to the pump beam, as expected. As the angle is increased, $\mathrm{THz}$ emission increases linearly, with negative peak-to-peak values representing a reversal in the polarity of the $\mathrm{THz}$ pulse. The DM emitter demonstrated strong emission at $0^{\circ}$ with no polarity inversion at other angles. This is attributed to the device emitting both from photoDember currents and the intrinsic net lateral current due to the Schottky effects. The emission asymmetry with angle is due to the asymmetric nature of LPD emission in general [18] and the asymmetrical geometry of the DM emitter element. A circular 


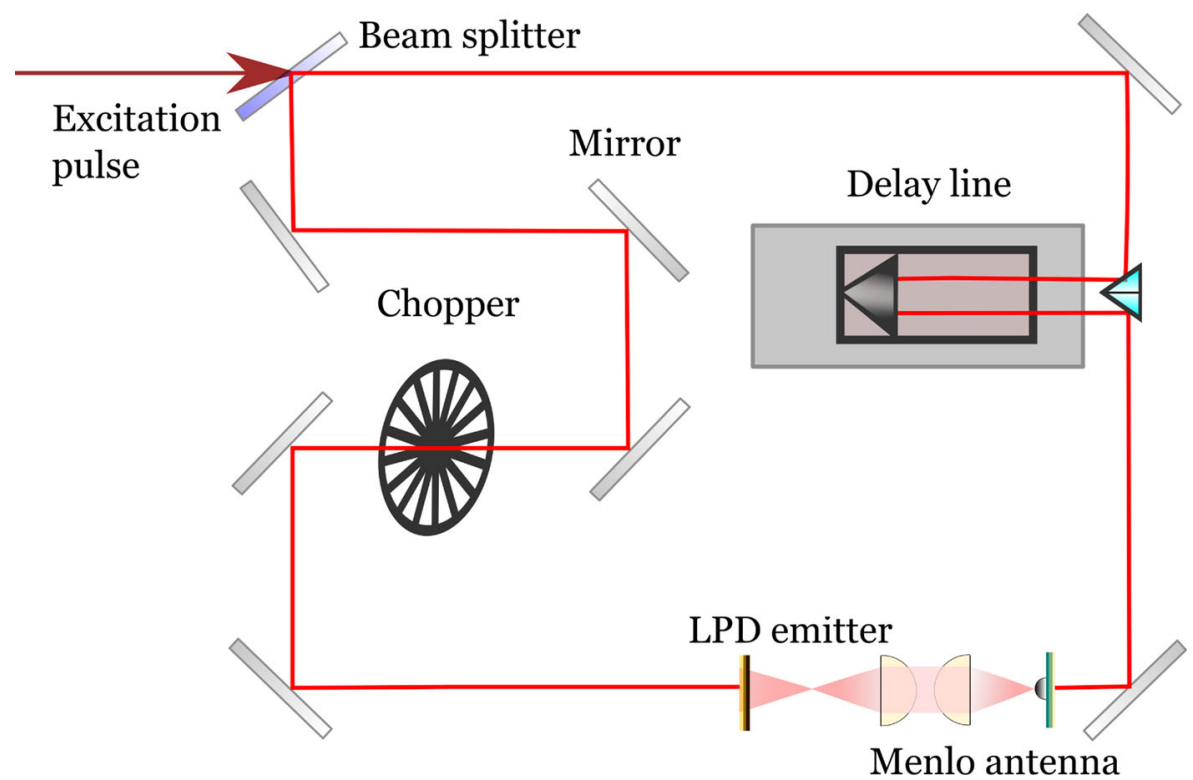

Fig. 1 A schematic of the experimental configuration can be seen. The emitter arm consists of a chopper wheel to optically modulate the pump beam for lock-in detection before it is directed on to the emitter. Following the emitter, two $\mathrm{THz}$ TPX lenses collect the $\mathrm{THz}$ radiation and bring it to focus on a Si lens aligned behind a PCA. On the delay arm, the probe beam is guided towards the PCA via an optical delay introduced using a mechanical delay stage

DM emitter was designed to produce radially polarized $\mathrm{THz}$ emission, which has been shown to efficiently couple into coaxial waveguides [37]. The design for this emitter is shown in Fig. 3a where $7 \mu \mathrm{m}$ wide overlapping rings of $\mathrm{Au}$ and $\mathrm{Pb}$ were fabricated on top of SI-GaAs over a period of $21 \mu \mathrm{m}$. TDS scans were taken over
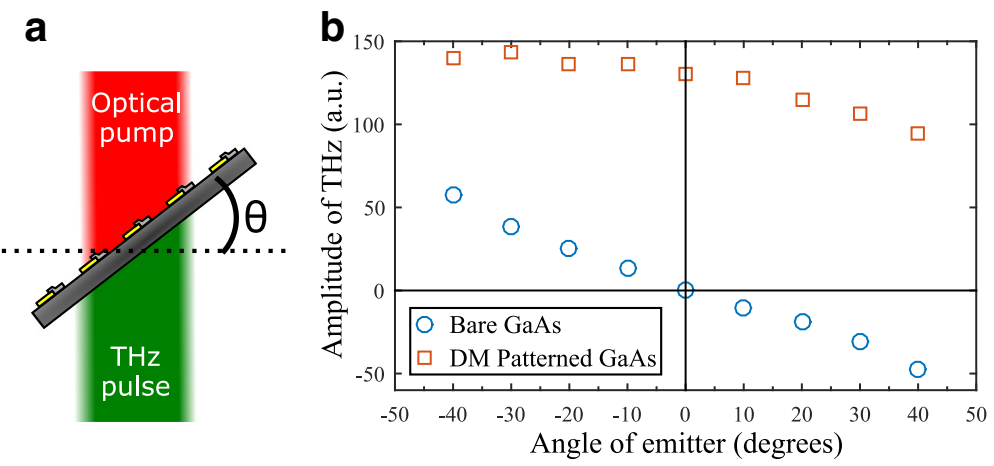

Fig. 2 Figure a shows a schematic for the angular measurements and defines the angle of emitter, $\theta$. Figure b shows a plot of the peak-to-peak amplitude of the emitted $\mathrm{THz}$ radiation with variation of the emitter angle in respect to the pump beam for an $\mathrm{Au} / \mathrm{Pb} \mathrm{DM}$ emitter (red squares) and bare SI-GaAs (blue circles). The DM emitter shows constant emission and no polarity flip compared to the bare SI-GaAs, which shows a polarity flip and no emission at $0^{\circ}$ as expected 
a

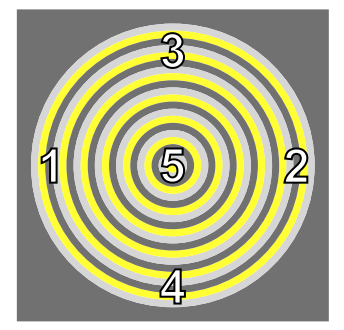

b

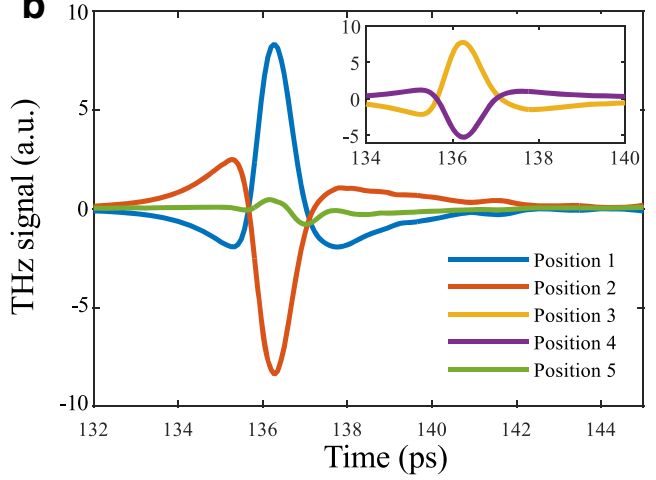

Fig. 3 a An illustration of the design of the circular DM emitter and the points illuminated. In $\mathbf{b}$, the results of TDS measurements taken at these respective points are shown. For opposing positions, the polarity of the detected THz is seen to flip. For positions 3 and 4 (inset), the emitter had to be rotated by $90^{\circ}$ to ensure the emitted polarization plane was in the same axis as the detector dipole so that a signal could be read. For the central position 5, around $10 \times$ less signal is recorded due to opposing polarizations cancelling in the far field

the areas indicated in Fig. 3a, and b shows the results. Illuminating opposite areas of the circular emitter produces $\mathrm{THz}$ with opposite polarity. For positions 3 and 4 , the emitter was rotated by $90^{\circ}$ so that the emitted polarization was in the same axis as the detector dipole. When we illuminate position 5, we show the resulting TDS scan taken when illuminating the centre of the circular emitter, which has a greatly reduced amplitude. In the far field, radial polarization cancels out and it is this effect that the THz-TDS system measures. In close proximity to, or butt-coupled to, with a dielectric coaxial waveguide, the radially polarized $\mathrm{THz}$ generated would be coupled into the waveguide efficiently without the need for launch optics, which are limited at $\mathrm{THz}$ frequencies.

\section{THz Focusing and Gouy Shift}

Here, we demonstrate control of the radiation of the THz LPD emitter with straight emitting elements by altering the characteristics of the input optical beam. We have placed the THz emitter between the lens that focuses the near IR laser beam and its focal point. Therefore, we transfer the properties of the excitation wavefront to the emitted $\mathrm{THz}$ wavefront to make the $\mathrm{THz}$ come to a focus at the point where the IR radiation would. In order for this to work, the radius of curvature of the wave has to be small enough to impose a temporal separation between the excitation of the centre and the edges of the emitter elements that is greater than the temporal width of the IR pump pulse. Further, the separation of emitter elements has to be much smaller than the emitted wavelength and the size of the emitter in total bigger. The experiment was initially performed using a titanium sapphire oscillator and consequently repeated with an amplifier to be able to extract frequency dependent data of the THz beam waists. Finally, a series of measurements that measures the Gouy phase 
shift was performed that confirmed that the $\mathrm{THz}$ emission undergoes focusing. For all these measurements, the straight element DM emitter was used that is described above.

\subsection{Titanium Sapphire Oscillator Pumped Focusing Experimental Results}

The emitter arm of the setup was changed from the one shown in Fig. 1 to resemble that shown in Fig. 4. The beam was expanded using a 13-mm focal length objective lens, collimated with a $5-\mathrm{cm}$ diameter and $100-\mathrm{mm}$ focal length lens, and then refocused across the $2 \times 2 \mathrm{~cm}$ DM emitter with an 87-mm focal length lens. After the emitter, two THz TPX lenses of $\mathrm{f}=60 \mathrm{~mm}$ were used to collect all of the emitted radiation, and then focus it upon the silicon lens aligned to behind the dipole of the detection emitter. The emitter can be translated anywhere between the $\mathrm{f}=87 \mathrm{~mm}$ focusing lens and the THz collecting lenses (a total translation distance of $83 \mathrm{~mm}$ due to mounts and holders). When the emitter is near the illuminating lens, the optical spot size is $2.36 \mathrm{~cm}$ in diameter and it excites roughly 870 strip emitters.

With the emitter at a fixed position of $37 \mathrm{~mm}$ behind the back of the focusing lens, knife edge measurements were taken at intervals between the emitter and the first THz TPX lens. At each position along the $z$-axis, where $z=0$ is defined as the point at the back plane of the emitter, a knife edge was used to occlude the beam and gradually reveal it by moving the knife in the $x$-direction. The $1 / \mathrm{e}^{2}$ spot size was measured from the lock-in amplifier readings and the results are shown in Fig. 5. The $\mathrm{THz}$ focus is then at approximately $87 \mathrm{~mm}$, as expected, by the focal length of the lens. The waist of the beam is approximately $1.3 \mathrm{~mm}$ but this is a measurement for the whole THz spectrum.

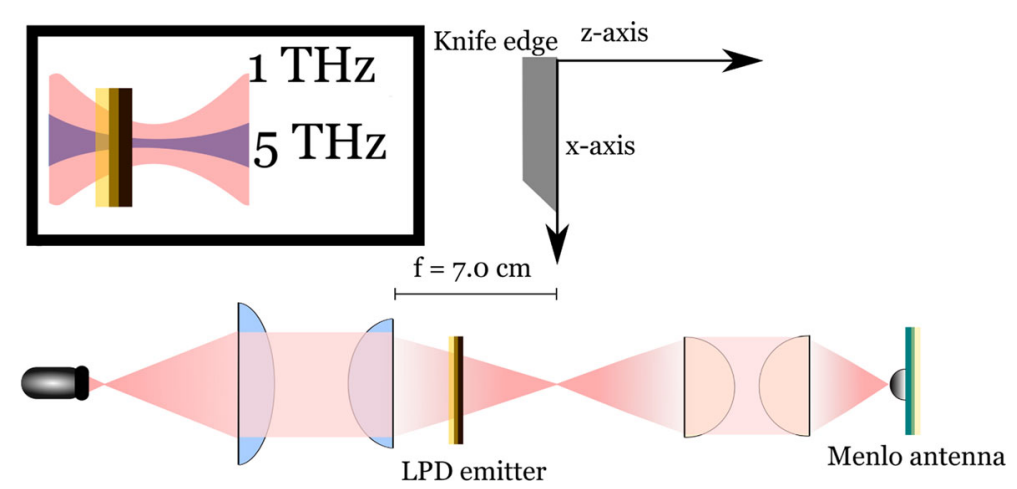

Fig. 4 The setup from Fig. 1 was modified by directing the pump beam through an objective lens and a 5-cm diameter collimator lens, and the beam was then incident upon an LPD emitter. Knife edge measurements of the focusing $\mathrm{THz}$ beam were performed with a sharp metallic blade at different positions in the $z$-direction, moving away from the LPD emitter. At each $z$ position, the knife was extended in the $x$ direction until the entire beam was occluded. The beam was then gradually revealed by moving the knife in the $x$-direction and time domain scans were recorded at each $x$ increment to provide the frequency content as a function of beam waist. Inset: The focusing qualities of the optical beam acquired from the focusing lens are expected to be transferred to the $\mathrm{THz}$ beam. The higher $\mathrm{THz}$ frequencies are expected to achieve a tighter focal spot than the lower frequencies 


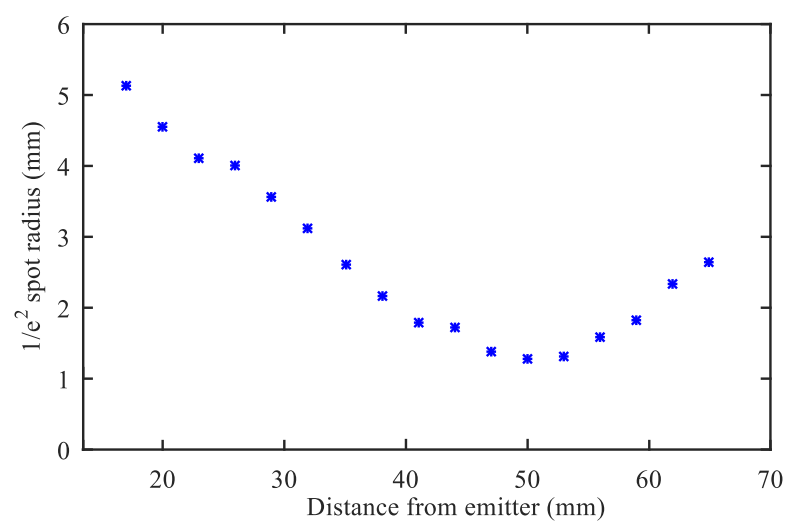

Fig. 5 The spot size against emitter distance of the LPD emitter using $400 \mathrm{~mW}$ irradiation from a titanium sapphire oscillator. The minimum spot size of the $\mathrm{THz}$ beam was measured to be $1.3 \mathrm{~mm}$ at a distance of $87 \pm 3 \mathrm{~mm}$ from the lens, matching closely the focal length of the lens

\subsection{Titanium Sapphire Amplifier Pumped Focusing Experimental Results}

Subsequently, the experiment was repeated with a titanium sapphire regenerative amplifier as the amplifier system has enough energy to saturate the large-area emitter and thus be able to extract frequency data. The amplifier provided $160 \mathrm{fs}$ pulses at a repetition rate of $250 \mathrm{KHz}$ and a peak pulse energy of approximately $5 \mu \mathrm{J}$. The only other change to the setup was to replace the focusing lens with a 70-mm focal length lens, the slightly sharper focusing lens was used in order to give a shorter radius of curvature to counterbalance for the longer pulse of the amplifier, and position the emitter at a fixed distance of $42 \mathrm{~mm}$ from the lens. The negative impact of using the amplifier is the relatively slow repetition rate $(\mathrm{kHz}$ instead of $\mathrm{MHz})$ that has a negative effect on the noise floor of the $\mathrm{THz}$ time domain scan and further the Ti:Sa amplifier has an asymmetric optical beam profile which made the results of the $\mathrm{THz}$ focusing more difficult to interpret. A measurement with a beam profiler of the laser amplifier beam is shown later in Fig. 7.

The knife edge measurements were performed for different distances from the emitter, which is $42 \mathrm{~mm}$ away from the lens, $z=0$ is defined as the point at the back plane of the emitter. At each point along the $z$-axis (see Fig. 4), a series of time domain scans were taken moving the knife in the $x$-direction to partially obscure the beam as described in Section 2. The expected behaviour of the intensity of the $\mathrm{THz}$ beam against $x$ position, assuming a Gaussian distribution, is expected to take the form of an error function [38].

A selection of beam waist profiles, for different frequencies, is displayed in Fig. 6 . The bandwidth provided by the LPD emitter, when considering the data taken for all $z$-positions, was insufficient to provide reliable analysis of individual wavelengths above $0.75 \mathrm{THz}$. In Fig. 6, the real data (points) and the fit at each of these $\mathrm{THz}$ frequencies can be seen. The fit curves were generated by fitting an error function to the normalized FFT data to extract the beam waist of the different frequencies to demonstrate that there is indeed focusing and that the higher frequencies reach a tighter focal 

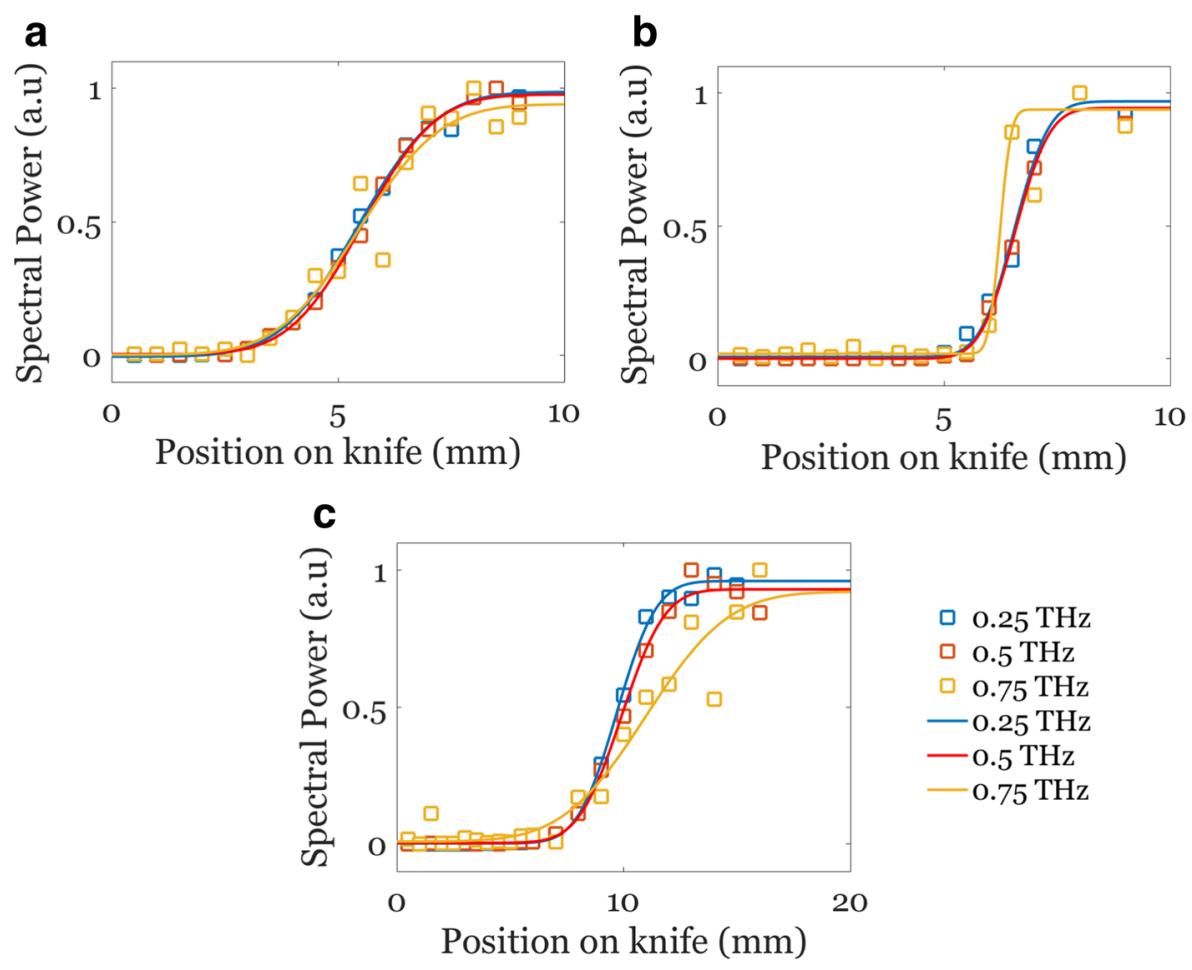

Fig. 6 The progression of the THz beam waist for different knife edge positions along the $z$-axis can be seen for distances of a $10 \mathrm{~mm} \mathrm{~b} 30 \mathrm{~mm}$ c) $39 \mathrm{~mm}$ from the back of the LPD emitter, where $\mathbf{b}$ is taken at the focus. The normalized real data is given as square points and the trend line is created using an error function

spot. The waists (as a function of $z$ ) can be seen to narrow and expand past the focus as expected if the $\mathrm{THz}$ beam is following the behaviour of the optical beam passing through a focusing lens. The focal point is near the $30 \mathrm{~mm}$ position, adding to this the $42 \mathrm{~mm}$ that is separating the emitter and the lens, the focus happens approximately $72 \mathrm{~mm}$ away from the lens which is close to its focal length $(70 \mathrm{~mm})$. The resulting waists at the $30-\mathrm{mm}$ position are, $0.85 \pm 0.2 \mathrm{~mm}$ at $0.25 \mathrm{THz}, 0.85 \pm 0.2 \mathrm{~mm}$ at $0.5 \mathrm{THz}$ and $0.28 \pm 0.1 \mathrm{~mm}$ at $0.75 \mathrm{THz}$. Using a very simple Gaussian approximation and the dimensions of our lenses, the waists should have been 1.1, 0.53 and $0.35 \mathrm{~mm}$. Our experiment is not characterized by an ideal Gaussian propagation, it is limited by the dynamic range of our measurements especially at $0.75 \mathrm{THz}$; the quality of the optical beam of the amplifier which is an asymmetric Gaussian (Fig. 7); the emission characteristics of the LPD DM emitters which—as they can be seen in Fig. 2b-are also asymmetric; finally, aperture effects on the lens system would make the beam non-Gaussian.

\subsection{Gouy Shift Experimental Results}

The Gouy shift for the LPD emitter was determined by taking FFTs as a function of emitter position on the $z$ axis (see Fig. 4), beginning with the emitter $42 \mathrm{~mm}$ from the 


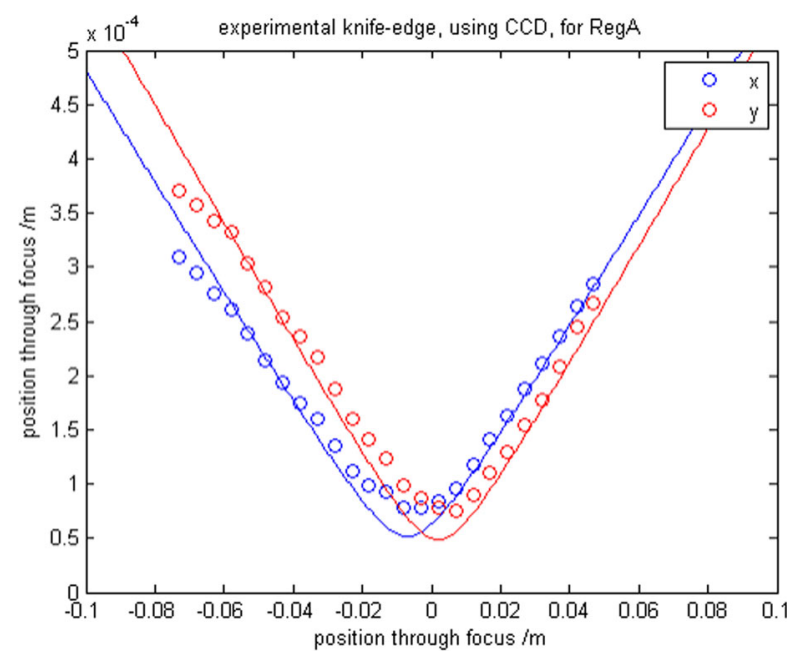

Fig. 7 This figure shows knife edge measurements of the beam profile of the pump IR beam as it comes from the regenerative titanium sapphire amplifier. The beam is asymmetric in $\mathrm{x}$ and $\mathrm{y}$ which complicates the propagation of the $\mathrm{THz}$ beam

IR focusing lens $(z=0)$ and gradually moving the THz emitter towards the THz TPX lenses. The Gouy shift was calculated for each position with respect to a reference waveform, which is defined as the complex FFT obtained when the emitter is $42 \mathrm{~mm}$ $(z=0 \mathrm{~mm})$ from the back of the focusing lens. The data had to be phase unwrapped to derive the relative phase; the phase unwrapping was performed from the FFT data after they were numerically smoothed by using a time window. It should be noted that the phase of the lock-in amplifier was not changed throughout the data taking.

The raw time domain data can be seen in Fig. 8a, where a polarity flip in the THz emission can be seen after the emitter is translated through the focus of the optical radiation. Examining Fig. 8a further, we see that the peak signal is positive and maximum when the emitter is closest to the lens (giving the trace in Fig. 8b) and when the emitter has passed the focal point, the opposite polarity is observed (Fig. 8c). This effect is due to the Gouy phase shift and it is visible due to the fact that we detect the E-field in the TDS setup rather than the average irradiance; both the electric field amplitude and phase are detected. Between these two points, the peak signal decreases until reaching the focus and then once again begins to increase after this point is passed; this is due to the size of the pump beam spot which illuminates fewer emitter elements near to the focus. On either side of the $\mathrm{THz}$ envelope peak in Fig. $8 \mathrm{~b}$ and $\mathrm{c}$ for each $z$ position fluctuations in the signal can be seen, due to etalon effects and other absorptions and reflections taking place within the setup. Such fluctuations cause aliasing of the data obtained in the main THz pulse and it is therefore desirable to reduce these as this will increase the accuracy of the subsequent phase unwrapping. Numerically, this can be done by applying a window function to the raw data that gradually reduces the signal to zero on either side of the main THz peak. Smoothing over a window is preferable as a sharp drop to zero of the time domain signal will cause errors and in this case, we used an exponential 

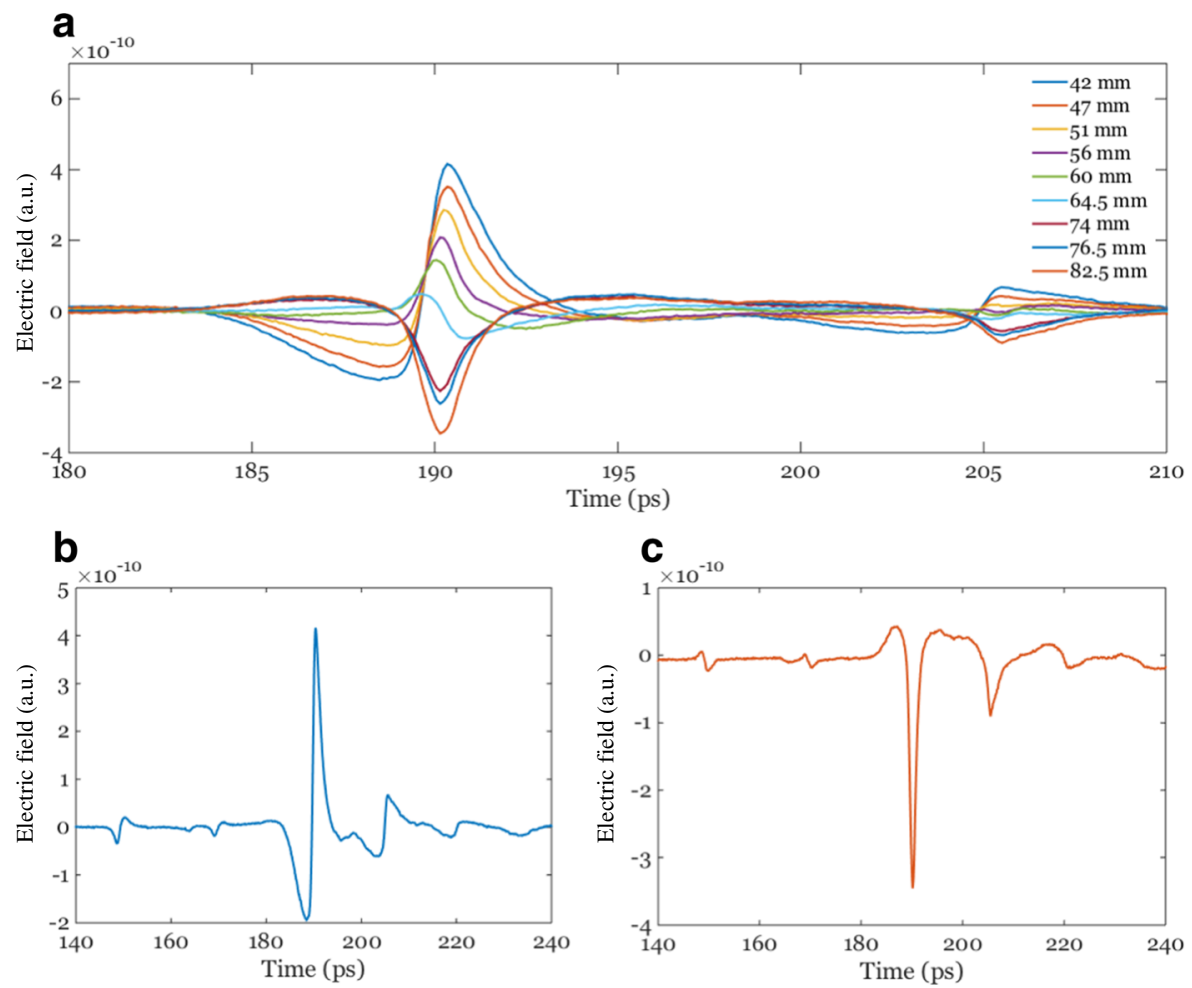

Fig. 8 The time domain data for each $z$ position can be seen in a. After the emitter translates through the focus of the optical beam the $\mathrm{THz}$ waveform undergoes a polarity switch as highlighted between $\mathbf{b}$ and c, where the emitter is at $42 \mathrm{~mm}(z=0 \mathrm{~mm})$ and $82.5 \mathrm{~mm}(z=40.5 \mathrm{~mm})$ behind the focusing lens, respectively. In a only a part of the time domain scan is shown, whereas in $\mathbf{b}$ and $\mathbf{c}$ the full time domain scan is shown

window function. With the smoothed FFT data obtained, the phase shift was extracted by taking the FFT at $42 \mathrm{~mm}$ as the reference point and subtracting all the subsequent phases of the FFTs from that point to obtain the relative shift in the data as a function of distance from the focusing lens. The results can be seen in Fig. 9 as a function of $\mathrm{THz}$ wavelength and show a dependence with frequency-the Rayleigh length is increasing as the frequency is decreasing - as expected. The equation for the Gouy phase; $A \cdot \arctan \left(z / z_{r}\right)$ was used for the fit, where $z$ is position along the beam, $z_{r}$ is the Rayleigh range, and $A$ is a scaling factor. The fits obtained by the Gouy phase shift data are better than the fits obtained by the waist experiment. This is expected because, in the case of the waist measurements, the beam is obscured by the razor blade which reduces the $\mathrm{THz}$ signal whereas in the case of the Gouy phase measurement, the whole $\mathrm{THz}$ beam is measured at all times. Also the phase and amplitude of the signal have different sources of noise in a THz-TDS experiment; the phase noise usually is dominated by the accuracy of the delay line whereas the amplitude is dominated by electrical and laser noise. The Rayleigh range is 4.6, 1.9 and $0.44 \mathrm{~mm}$ for $0.25,0.5$ and $0.75 \mathrm{THz}$, respectively. The corresponding calculated waists assuming Gaussian propagation is $1.3 \pm 0.1,0.85 \pm 0.1$ and $0.4 \pm 0.1 \mathrm{~mm}$ 


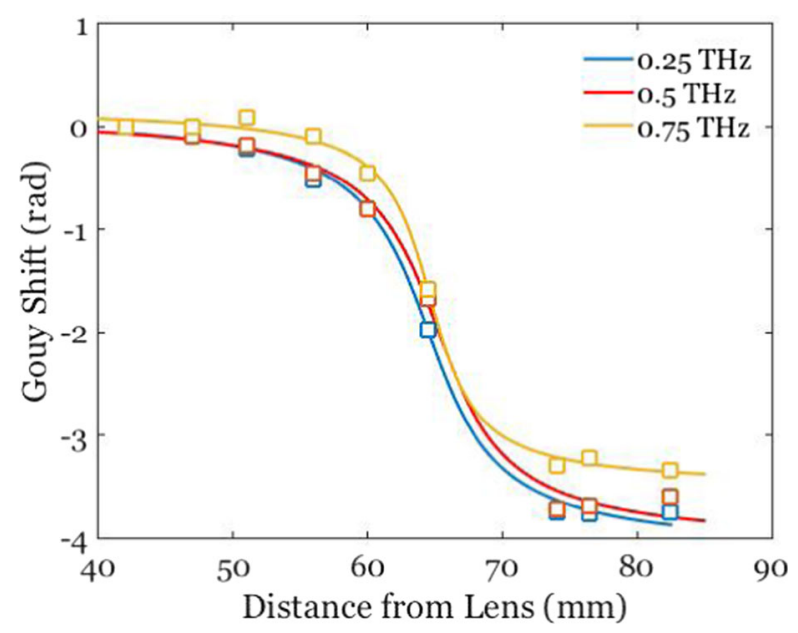

Fig. 9 The Gouy shift for $0.25,0.5$ and $0.75 \mathrm{THz}$ can be seen along with the trend lines using $A \cdot \arctan \left(z / z_{r}\right)$ for the fit

for $0.25,0.5$ and $0.75 \mathrm{THz}$, respectively. The estimated waists imply tight focusing as they are quite close to the wavelengths $(1.2,0.6$ and $0.4 \mathrm{~mm}$ for $0.25,0.5$ and $0.75 \mathrm{THz}$, respectively) and they are comparable to the results shown in Fig. 6.

\section{Conclusion}

The THz emitted from the LPD multiplexed strip emitters was brought to a focus by transferring the wavefront characteristics of the focusing Ti:Sa excitation pulse on to the emitter. The data provided by the knife edge measurements shows that the $\mathrm{THz}$ wavefronts take on the characteristics of the optical pulse and can be used for beam focusing and in the future beam steering if a spatial light modulator is used in the path of the optical pumping beam. This way, an arbitrary pattern of $\mathrm{THz}$ radiation could be produced in order to speed up imaging applications. We also demonstrated that the $\mathrm{THz}$ beam experiences a Gouy shift which provides further evidence of focusing and introduces a way to generate a selective polarity flip of the $\mathrm{THz}$ radiation if it is experimentally required by simply moving the emitter. We also show that $\mathrm{THz} \mathrm{DM}$ emitters can be fabricated following a circular pattern to create $\mathrm{THz}$ with radially symmetric polarization, which is required for launching $\mathrm{THz}$ on to a coaxial wire guide. The combination of the presented techniques would allow for emitters to be designed with a waveguide mode in mind, with the $\mathrm{THz}$ emission steered and focused into the waveguide by manipulating the pump beam for efficient coupling.

We would like to acknowledge EPSRC for support under grant EP/J007676/1, "Quantum Cascade amplifiers for high power Terahertz time domain spectrometry". All data supporting this study are openly available from the University of Southampton repository, in https://doi.org/10.5258/SOTON/D0289. 
Open Access This article is distributed under the terms of the Creative Commons Attribution 4.0 International License (http://creativecommons.org/licenses/by/4.0/), which permits unrestricted use, distribution, and reproduction in any medium, provided you give appropriate credit to the original author(s) and the source, provide a link to the Creative Commons license, and indicate if changes were made.

\section{References}

1. Y. Monnai, K. Altmann, C. Jansen, H. Hillmer, M. Koch, H. Shinoda, Optics Express 21(2), 2347 (2013). https://doi.org/10.1364/OE.21.002347. https://www.osapublishing.org/oe/abstract.cfm? uri=oe-21-2-2347.

2. N. Krumbholz, K. Gerlach, F. Rutz, M. Koch, R. Piesiewicz, T. Kürner, D. Mittleman, Applied Physics Letters 88(20), 202905 (2006). https://doi.org/10.1063/1.2205727. http://scitation.aip.org/content/aip/ journal/apl/88/20/10.1063/1.2205727.

3. R. Piesiewicz, T. Kleine-Ostmann, N. Krumbholz, D. Mittleman, M. Koch, J. Schoebei, T. Kurner, IEEE Antennas and Propagation Magazine 49(6), 24 (2007). https://doi.org/10.1109/MAP.2007. 4455844.

4. K.L. Wang, D.M. Mittleman, Nature 432(7015), 376 (2004). https://doi.org/10.1038/nature03040.

5. O. Mitrofanov, T. Tan, P.R. Mark, B. Bowden, J.A. Harrington, Applied Physics Letters 94(17), 171104 (2009). https://doi.org/10.1063/1.3126053. http://scitation.aip.org/content/aip/journal/apl/94/ $17 / 10.1063 / 1.3126053$.

6. S. Busch, B. Scherger, M. Scheller, M. Koch, Optics Letters 37(8), 1391 (2012). https://doi.org/10. 1364/OL.37.001391. https://www.osapublishing.org/abstract.cfm?URI=ol-37-8-1391.

7. K.i. Maki, C. Otani, Optics Express 16(14), 10158 (2008). https://doi.org/10.1364/OE.16.010158. https://www.osapublishing.org/oe/abstract.cfm?uri=oe-16-14-10158.

8. J. O'Hara, D. Grischkowsky, Journal of the Optical Society of America B 21(6), 1178 (2004). https:// doi.org/10.1364/JOSAB.21.001178. https://www.osapublishing.org/abstract.cfm?URI=josab-21-61178.

9. J. Hebling, G. Almasi, I. Kozma, J. Kuhl, Optics Express 10(21), 1161 (2002). https://doi.org/10.1364/ OE.10.001161. https://www.osapublishing.org/oe/abstract.cfm?uri=oe-10-21-1161.

10. C. Weiss, G. Torosyan, J.P. Meyn, R. Wallenstein, R. Beigang, Y. Avetisyan, Optics Express 8(9), 497 (2001). https://doi.org/10.1364/OE.8.000497. https://www.osapublishing.org/abstract.cfm? URI=oe-8-9-497.

11. A. Eshaghi, M. Shahabadi, L. Chrostowski, Journal of the Optical Society of America B 29(4), 813 (2012). https://doi.org/10.1364/JOSAB.29.000813. https://www.osapublishing.org/abstract.cfm? URI=josab-29-4-813.

12. K. Uematsu, K.i. Maki, C. Otani, Optics Express 20(20), 22914 (2012). https://doi.org/10.1364/OE. 20.022914. https://www.osapublishing.org/oe/abstract.cfm?uri=oe-20-20-22914.

13. B.B. Hu, J.T. Darrow, X.C. Zhang, D.H. Auston, P.R. Smith, Applied Physics Letters 56(10), 886 (1990). https://doi.org/10.1063/1.102618. http://scitation.aip.org/content/aip/journal/apl/56/10/ $10.1063 / 1.102618$.

14. N.M. Froberg, B.B. Hu, X.C. Zhang, D.H. Auston, IEEE Journal of Quantum Electronics 28(10), 2291 (1992). https://doi.org/10.1109/3.159536.

15. N. Froberg, M. Mack, B.B. Hu, X.C. Zhang, D.H. Auston, Applied Physics Letters 58(5), 446 (1991). https://doi.org/10.1063/1.104629. http://scitation.aip.org/content/aip/journal/apl/58/5/10. 1063/1.104629.

16. B.B. Hu, N. Froberg, M. Mack, X.C. Zhang, D.H. Auston, Applied Physics Letters 58(13), 1369 (1991). https://doi.org/10.1063/1.104311. http://scitation.aip.org/content/aip/journal/apl/58/13/ 10.1063/1.104311.

17. M.E. Barnes, D. McBryde, G.J. Daniell, G. Whitworth, A.L. Chung, A.H. Quarterman, K.G. Wilcox, A. Brewer, H.E. Beere, D.A. Ritchie, V. Apostolopoulos, Optics Express 20(8), 8898 (2012). https://doi.org/10.1364/OE.20.008898. https://www.osapublishing.org/oe/abstract.cfm? uri=oe-20-8-8898.

18. M.E. Barnes, S.A. Berry, P. Gow, D. McBryde, G.J. Daniell, H.E. Beere, D.A. Ritchie, V. Apostolopoulos, Optics Express 21(14), 16263 (2013). https://doi.org/10.1364/OE.21.016263. https:// www.osapublishing.org/abstract.cfm?URI=oe-21-14-16263. 
19. G. Klatt, F. Hilser, W. Qiao, M. Beck, R. Gebs, A. Bartels, K. Huska, U. Lemmer, G. Bastian, M. Johnston, M. Fischer, J. Faist, T. Dekorsy, Optics Express 18(5), 4939 (2010). https://doi.org/10.1364/OE. 18.004939. http://www.opticsinfobase.org/oe/abstract.cfm?uri=oe-18-5-4939.

20. D. McBryde, P. Gow, S.A. Berry, A. Aghajani, M.E. Barnes, V. Apostolopoulos, Applied Physics Letters 104(20), 201108 (2014). https://doi.org/10.1063/1.4878739.http://arxiv.org/abs/1404.5792.

21. P. Gow, D. McBryde, S. Berry, M. Barnes, V. Apostolopoulos, Electronics Letters 50(25), 1966 (2014). https://doi.org/10.1049/el.2014.2751.

22. M. Nikfalazar, C. Kohler, A. Wiens, A. Mehmood, M. Sohrabi, H. Maune, J.R. Binder, R. Jakoby, IEEE Microwave and Wireless Components Letters 26(1), 70 (2016). https://doi.org/10.1109/LMWC. 2015.2505633.

23. T. Tyc, Optics Letters 37(5), 924 (2012). https://doi.org/10.1364/OL.37.000924. https://www. osapublishing.org/abstract.cfm?URI=ol-37-5-924.

24. M.S. Kim, T. Scharf, A.d.C. Assafrao, C. Rockstuhl, S.F. Pereira, H.P. Urbach, H.P. Herzig, Optics Express 20(27), 28929 (2012). https://doi.org/10.1364/OE.20.028929. https://www.osapublishing. org/oe/abstract.cfm?uri=oe-20-27-28929.

25. R.L. Nowack, S.M. Kainkaryam, Geophysical Journal International 184(2), 965 (2011). https://doi.org/10.1111/j.1365-246X.2010.04900.x.http://gji.oxfordjournals.org/content/184/2/965.

26. R. Borghi, M. Santarsiero, R. Simon, Journal of the Optical Society of America A 21(4), 572 (2004). https://doi.org/10.1364/JOSAA.21.000572. https://www.osapublishing.org/abstract.cfm? URI=josaa-21-4-572.

27. A.B. Ruffin, J.V. Rudd, J.F. Whitaker, S. Feng, H.G. Winful, Physical Review Letters 83(17), 3410 (1999). https://doi.org/10.1103/PhysRevLett.83.3410.http://link.aps.org/doi/10.1103/ PhysRevLett.83.3410.

28. R.W. McGowan, R.A. Cheville, D. Grischkowsky, Applied Physics Letters 76(6), 670 (2000). https:// doi.org/10.1063/1.125857. http://scitation.aip.org/content/aip/journal/apl/76/6/10.1063/1.125857.

29. K.J. Kaltenecker, J.C. König-Otto, M. Mittendorff, S. Winnerl, H. Schneider, M. Helm, H. Helm, M. Walther, B.M. Fischer, Optica 3(1), 35 (2016). https://doi.org/10.1364/OPTICA.3.000035. https:// www.osapublishing.org/abstract.cfm?URI=optica-3-1-35.

30. S. Feng, H.G. Winful, Journal of the Optical Society of America A 17(11), 2096 (2000). https://doi. org/10.1364/JOSAA.17.002096. https://www.osapublishing.org/abstract.cfm?URI=josaa-17-11-2096.

31. S. Feng, H.G. Winful, R.W. Hellwarth, Optics Letters 23(5), 385 (1998). https://doi.org/10.1364/OL. 23.000385. https://www.osapublishing.org/abstract.cfm?URI=ol-23-5-385.

32. M. Yi, K. Lee, J.D. Song, J. Ahn, Applied Physics Letters 100(16), 161110 (2012). https://doi.org/10.1063/1.4705294. http://scitation.aip.org/content/aip/journal/apl/100/16/10.1063/1. 4705294.

33. L. Zhang, H. Zhong, K. Mu, C. Zhang, Y. Zhao, Optics Express 20(1), 75 (2012). https://doi.org/10. 1364/OE.20.000075. https://www.osapublishing.org/oe/abstract.cfm?uri=oe-20-1-75.

34. H. Chen, Q. Zhan, Y. Zhang, Y.P. Li, Physics Letters A 371(3), 259 (2007). https://doi.org/10.1016/j. physleta.2007.03.095. http://www.sciencedirect.com/science/article/pii/S0375960107004501.

35. H. He, X.C. Zhang, Applied Physics Letters 100(6), 061105 (2012). https://doi.org/10.1063/1.368 2517. http://scitation.aip.org/content/aip/journal/apl/100/6/10.1063/1.3682517.

36. S. Ahmed, J. Savolainen, P. Hamm, Optics Express 22(4), 4256 (2014). https://doi.org/10.1364/OE. 22.004256. https://www.osapublishing.org/oe/abstract.cfm?uri=oe-22-4-4256.

37. M. Navarro-Cía, J. Wu, H. Liu, O. Mitrofanov, in 201641 st International Conference on Infrared, Millimeter, and Terahertz waves (IRMMW-THz), (IEEE, 2016), pp. 1-2.

38. A. Dobroiu, M. Yamashita, Y.N. Ohshima, Y. Morita, C. Otani, K. Kawase, Applied Optics 43(30), 5637 (2004). https://doi.org/10.1364/AO.43.005637. https://www.osapublishing.org/abstract. cfm?URI=ao-43-30-5637. 